\title{
SYNTHESIS AND CHARACTERIZATION OF NOVEL 1,3-THIAZOLE AND 2-AMINO-1,3,4-THIADIAZOLE DERIVATIVES
}

\author{
Mustafa Er, Ayşe Şahin, Hakan Tahtaci \\ Department of Chemistry, Karabuk University, 78050 Karabuk, Turkey \\ mustafaer@karabuk.edu.tr
}

\begin{abstract}
Thiosemicarbazone derivatives 3a-e were synthesized by the reaction of various aldehydes $1 \mathbf{1 a}-\mathbf{e}$ with 4-methyl thiosemicarbazide $\mathbf{2}$ in $78 \%$ to $90 \%$ yield. Then, the thiazole moieties of the target materials $\mathbf{5 a}-\mathbf{e}$ were obtained in high yields $(71-93 \%)$ using the Hantzsch reaction utilizing thiosemicarbazone derivatives 3a-e with ethyl-2-chloroacetoacetic ester. The substituted nitrile derivatives $7 \mathbf{a}-\mathbf{e}$ were obtained in moderate to high yield (58-84\%) from the reaction of compounds 5a-e with chloroacetonitrile by the nucleophilic aliphatic substitution reaction in the presence of anhydrous potassium carbonate. Finally, substituted 2-amino-1,3,4-thiadiazole compounds 9a-e were obtained in moderate to good yields (51-62\%) from the reaction of thiosemicarbazide with substituted nitrile derivatives 7a-e. As a result, compounds that all share a high disposition for biological activities were obtained. The structures of the newly synthesized compounds were confirmed by IR, ${ }^{1} \mathrm{H}$ NMR, ${ }^{13} \mathrm{C}$ NMR, elemental analysis, and mass spectrometric techniques.
\end{abstract}

Keywords: thiosemicarbazone; 1,3-thiazole; 2-amino-1,3,4-thiadiazole; Hantzsch reaction

\section{СИНТЕЗА И КАРАКТЕРИЗАЦИЈА НА НОВИ ДЕРИВАТИ НА 1,3-ТИАЗОЛ И 2-АМИНО-1,3,4-ТИАДИАЗОЛ}

Тиосемикарбазонските деривати 3a-e беа синтетизирани при реакција на разни алдехиди 1a-e co 4-метилтиосемикарбазид 2 со принос од 78 до 90\%. Тиазолските прстени на целните соединенија 5а-е беа добиени со висок принос од 71-93\% со реакцијата на Hantzsch со примена на тиосемикарбазонските деривати $\mathbf{3 a - e}$ и етил-2-хлороацетооцетен естер. Супституираните нитрилни деривати 7а-e беа добиени со умерен до висок принос при реакција на соединенијата 5а-e со хлороацетонитрил при нуклеофилна алифатична супституција во присуство на безводен калиумкарбонат. Конечно, супституираните 2-амино-1,3,4-тиодиазолните соединенија 9а-е беа добиени со умерен до добар принос (51-62\%) при реакција на тиосемикарбазид со супституирани нитрилни деривати 7a-e. Сите овие добиени соединенија покажуваат висока биолошка активност. Структурите на новосинтетизираните соединенија беа потврдени по пат на IR, ${ }^{1} \mathrm{H} \mathrm{NMR},{ }^{13} \mathrm{C} \mathrm{NMR}$, елементарна анализа и масеноспектрометриски техники.

Клучни зборови: тиосемикарбазон; 1,3-тиазол; 2-амино-1,3,4-тиадиазол; реакција на Hantzsch

\section{INTRODUCTION}

In recent years, despite the discovery and analysis of an increasing number of compounds exhibiting biological properties, their usage has been limited due to application difficulties, the emergence of drug resistance, unwanted side effects, and pharmacokinetic deficiencies. Thus, great efforts have been made by chemists to ana- lyze and synthesize compounds exhibiting biological activities that have the potential to be used in medicinal chemistry. Thiazoles, thiadiazoles, and their heterocyclic derivatives have attracted continuing interest over the years because of their varied biological properties [1-3].

Thiazole and thiadiazole derivatives are known to exhibit various biological activities, such as anti-allergy [4], antihypertensive [5], anti-in- 
flammatory [6-7], anti-schizophrenia [8], antifungal [9], antibacterial [10], antimicrobial [11-13], analgesic [14], anti-HIV [15], anticonvulsant [16], antidepressant [17], and anti-cancer [18-21] properties. Other uses of heterocyclic compounds containing nitrogen and sulfur include manufacturing biocides, dyes [22-24], and plant-growth regula- tors [25]. Thus, the synthesis of thiazole and thiadiazole derivatives is currently of great importance to the scientific community.

The purpose of the present study was to obtain and characterize novel thiosemicarbazone, 1,3thiazole, nitrile, and 2-amino-1,3,4-thiadiazole derivatives (Scheme 1).<smiles>[Y]c1cc(C=O)cc([X])c1O</smiles><smiles>[X]c1cc(/C=N/NC(=S)NC)cc([X])c1O</smiles><smiles>C/C=C\C=N\NC(=S)NC</smiles><smiles>CCOC(=O)C(Cl)C(C)=O</smiles><smiles>CCOC(=O)C1S/C(=N/N=C/c2ccccc2O)N(C)C1C</smiles>

$5 e$<smiles>[X]c1cc(/C=N/N=c2/sc(C(=O)OC)c(C)n2C)cc([Y])c1O</smiles><smiles>[Y]c1cc(/C=N/N=c2sc(C(=O)OCC)c(C)n2C)cc([X])c1OCC#N</smiles><smiles>COC(=O)c1s/c(=N\N=C\c2ccccc2OCc2nnc(N)s2)n(C)c1C</smiles><smiles>C=C1CC1C</smiles>

i: $140-160^{\circ} \mathrm{C}$, without solvent

ii: Absolute ethyl alcohol, reflux, 3 days iii: Dry acetone, dry $\mathrm{Na}_{2} \mathrm{CO}_{3}$, reflux, 2 days

iv: Trifluoroacetic acid (TFA), $60 \stackrel{\circ}{\circ}$, reflux, 5 hours<smiles>[X]c1cc(/C=N/N=c2/sc(C(=O)OC)c(C)n2C)cc([X])c1OCc1nnc(N)s1</smiles>

Scheme 1. Synthetic pathway for the preparation of target compounds $(\mathbf{3}, \mathbf{5}, \mathbf{7}, \mathbf{9} \mathbf{a}-\mathbf{e})$. 


\section{EXPERIMENTAL}

\section{Materials and methods}

Melting points were recorded on a Gallenkamp melting point apparatus and were uncorrected. ${ }^{1} \mathrm{H}$ NMR and ${ }^{13} \mathrm{C}$ NMR spectra were recorded on a Varian-Mercury $200 \mathrm{MHz}$ spectrometer. The IR spectra were measured in potassium bromide pellets using a Perkin-Elmer 1600 series FTIR spectrometer. The mass spectra were measured with a Micromass Quattro LC/ULTIMA LCMS/MS spectrometer in the positive ion mode using pyridine-methanol as a solvent. Elemental analysis was performed on a Hewlett-Packard 185 $\mathrm{CHN}$ analyzer. Elemental values of the synthesized compounds agreed with the calculated ones. All of the chemicals were obtained from Fluka Chemie AG Buchs (Switzerland). Compounds 3d [26], 3e [27], 5d [26], and 5e [27] have been published.

\section{General method for the synthesis of thiosemicarbazone derivatives $(3 a-c)$}

In a round-bottomed flask, compounds 1a-c $(0.0125 \mathrm{~mol})$ and 4-methyl thiosemicarbazide 2 $(0.0125 \mathrm{~mol})$ were heated to $160^{\circ} \mathrm{C}$ without solvent in an oil bath and stirred for $4 \mathrm{~h}$. Dimethylformamide (DMF) was added to the reaction and dissolved. Water was then added to the solution and a solid precipitated. The solution was filtered, and the solid was washed with ethanol. The precipitated solid was recrystallized from an appropriate solvent to afford the desired compound.

1-(4-Hydroxybenzylidene)-4-methylthiosemicarbazide (3a). The solid obtained was washed with $\mathrm{H}_{2} \mathrm{O}$ and recrystallized (yield: $2.04 \mathrm{~g}, 78 \%$ ); white solid, mp $224-225^{\circ} \mathrm{C}$ (from EtOH-water, 1:1) IR $(\mathrm{KBr})\left(v_{\max }, \mathrm{cm}^{-1}\right), 3342(\mathrm{OH}), 3178(\mathrm{NH}), 3009$ (Ar-CH), 2936 (Aliph. CH), $1599(\mathrm{C}=\mathrm{N}), 1562$ $(\mathrm{C}=\mathrm{C}) .{ }^{1} \mathrm{H}$ NMR $(200 \mathrm{MHz}$, DMSO-d 6 ) $\delta(\mathrm{ppm})$, 2.98-3.00 (d, 3H, N-CH 3 ), Ar-H [6.76-6.80 (d, $2 \mathrm{H}), 7.59-7.63(\mathrm{~d}, 2 \mathrm{H})$ ], $7.94(\mathrm{~s}, 1 \mathrm{H}, \mathrm{CH}=\mathrm{N}), 8.36$, $\left(\mathrm{s}, 1 \mathrm{H}, \mathrm{NH}-\mathrm{CH}_{3}\right), 9.88,(\mathrm{~s}, 1 \mathrm{H}, \mathrm{OH}), 11.26,(\mathrm{~s}, 1 \mathrm{H}$, $\mathrm{NH}) .{ }^{13} \mathrm{C}$ NMR (100 MHz, DMSO-d 6 ) $\delta(\mathrm{ppm})$, 30.66, 115.44, 125.15, 128.86, 159.06, 142.04, 177.22. MS(ESI- $m / z):(M+1)^{+}: 210.39$. Anal. for $\mathrm{C}_{9} \mathrm{H}_{11} \mathrm{~N}_{3} \mathrm{OS}$ (Mw 209.27). Found \% C 51.68; $\mathrm{H}$ 5.24; N 20.14. Calculated \% C 51.65; H 5.30; N 20.08 .

1-(4-Hydroxy-3-methoxybenzylidene)-4-methylthiosemicarbazide (3b). The solid obtained was washed with $\mathrm{H}_{2} \mathrm{O}$, alcohol and recrystallized (yield: $2.48 \mathrm{~g}, 83 \%$ ); yellow solid, $\mathrm{mp} 195-196^{\circ} \mathrm{C}$ (from DMF-EtOH, 1:1) IR (KBr) $\left(v_{\max }, \mathrm{cm}^{-1}\right)$,
$3358(\mathrm{OH}), 3147(\mathrm{NH}), 2999(\mathrm{Ar}-\mathrm{CH}), 2954$ (Aliph. CH), $1599(\mathrm{C}=\mathrm{N}), 1553(\mathrm{C}=\mathrm{C}) .{ }^{1} \mathrm{H}$ NMR (200 MHz, DMSO-d $\left.\mathrm{d}_{6}\right) \delta(\mathrm{ppm}), 3.01-3.03(\mathrm{~d}, 3 \mathrm{H}$, $\left.\mathrm{N}-\mathrm{CH}_{3}\right), 3.83\left(\mathrm{~s}, 3 \mathrm{H},\left(\mathrm{OCH}_{3}\right)\right)$, Ar-H [6.77-6.80 (d, $1 \mathrm{H}), 7.07-7.12(\mathrm{~d}, 1 \mathrm{H}), 7.40(\mathrm{~s}, 1 \mathrm{H})], 7.93,(\mathrm{~s}, 1 \mathrm{H}$, $\mathrm{CH}=\mathrm{N}), 8.35-8.37,\left(\mathrm{~d}, 1 \mathrm{H}, \mathrm{NH}-\mathrm{CH}_{3}\right), 9.47,(\mathrm{~s}, 1 \mathrm{H}$, $\mathrm{OH}), 11.30,(\mathrm{~s}, 1 \mathrm{H}, \mathrm{NH}) .{ }^{13} \mathrm{C} \mathrm{NMR}(100 \mathrm{MHz}$, DMSO-d $\left._{6}\right) \delta$ (ppm), 30.70, 55.70, 109.66, 115.24, 121.87, 127.54, 142.37, 147.90, 148.63, 177.22. MS(ESI- $m / z): \quad(\mathrm{M}+1)^{+}: \quad 240.14$. Anal. for $\mathrm{C}_{10} \mathrm{H}_{13} \mathrm{~N}_{3} \mathrm{O}_{2} \mathrm{~S}$ (Mw 239.29). Found \% C 50.27; $\mathrm{H}$ 5.34; N 17.49. Calculated \% C 50.19; H 5.48; N 17.56.

1-(4-Hydroxy-3,5-dimethylbenzylidene)-4-methylthiosemicarbazide (3c). The solid obtained was washed with $\mathrm{H}_{2} \mathrm{O}$ and recrystallized (yield: $2.70 \mathrm{~g}$, 90\%); white solid, mp $214-215^{\circ} \mathrm{C}$ (from EtOH) IR $(\mathrm{KBr})\left(v_{\max }, \mathrm{cm}^{-1}\right), 3100-3450(\mathrm{OH}), 3165(\mathrm{NH})$, 3001 (Ar-CH), 2971 (Aliph. CH), $1591(\mathrm{C}=\mathrm{N})$, $1483(\mathrm{C}=\mathrm{C}) .{ }^{1} \mathrm{H}$ NMR (200 MHz, DMSO-d $\left.{ }_{6}\right) \delta$ (ppm), 2.17 (s, 6H, $\mathrm{CH}_{3}$ ), 2.99 (s, 3H, N-CH $\mathrm{CH}_{3}$, Ar$\mathrm{H}[7.34(\mathrm{~s}, 2 \mathrm{H})], 7.88(\mathrm{~s}, 1 \mathrm{H}, \mathrm{CH}=\mathrm{N}), 8.74(\mathrm{bs}, 1 \mathrm{H}$, $\left.\mathrm{NH}_{-} \mathrm{CH}_{3}\right), 8.79(\mathrm{~s}, 1 \mathrm{H}, \mathrm{OH}), 11.32(\mathrm{~s}, 1 \mathrm{H}, \mathrm{NH}) .{ }^{13} \mathrm{C}$ NMR (100 MHz, DMSO-d $\left.{ }_{6}\right) \delta$ (ppm), 17.25, $31.45,125.10,125.73,128.25,143.20,155.87$, 177.84. MS(ESI- $m / z)$ : $(\mathrm{M}+1)^{+}: 238.02$; Anal. for $\mathrm{C}_{11} \mathrm{H}_{15} \mathrm{~N}_{3} \mathrm{OS}$ (Mw 237.32). Found \% C 55.55; $\mathrm{H}$ 6.41; N 17.75. Calculated \% C 55.67; H 6.37; N 17.71 .

General method for the synthesis of 1,3-thiazole derivatives $(5 a-c)$

In a two-necked flask, compounds 3a-c $(0.009 \mathrm{~mol})$ were suspended in absolute ethanol. The solution of compound $4(0.009 \mathrm{~mol})$ in absolute ethanol was then dropped into the suspension for $30 \mathrm{~min}$. The reaction mixture was refluxed for 3 days and the clear color of the mixture turned to yellow. The crude product was filtrated and washed with water. The precipitated solid was recrystallized from an appropriate solvent to afford the desired compound.

(E)-Ethyl 2-(2-(4-hydroxybenzylidene)hydrazono)-3,4-dimethyl-2,3-dihydrothiazole-5-carboxylate (5a). The solid was recrystallized (yield: 2.67 $\mathrm{g}, 93 \%$ ); white crystals, $\mathrm{mp} 289-290^{\circ} \mathrm{C}$ (from DMF-EtOH- $\left.\mathrm{H}_{2} \mathrm{O}, 1: 4: 1\right)$ IR $(\mathrm{KBr})\left(v_{\max }, \mathrm{cm}^{-1}\right)$, 3150-3450 (OH), 3026 (Ar-CH), 2980 (Aliph. $\mathrm{CH}), 1700(\mathrm{C}=\mathrm{O}), 1603(\mathrm{C}=\mathrm{N}), 1278,1262,1234$ (C-O-C), $1084\left(\mathrm{OCH}_{2}-\mathrm{CH}_{3}\right) .{ }^{1} \mathrm{H}$ NMR $(200 \mathrm{MHz}$, DMSO-d $\left._{6}\right) \delta(\mathrm{ppm}), 1.20-1.28$ (t, 3H, Ester- $\mathrm{CH}_{3}$ ), $2.52\left(\mathrm{~s}, 3 \mathrm{H}\right.$, Thiazole- $\left.\mathrm{CH}_{3}\right), 3.38\left(\mathrm{~s}, 3 \mathrm{H}, \mathrm{N}-\mathrm{CH}_{3}\right)$, 4.14-4.21 (q, 2H, Ester-OCH ${ }_{2}$ ), Ar-H [6.79-6.84 $(\mathrm{d}, 2 \mathrm{H}), 7.54-7.59(\mathrm{~s}, 2 \mathrm{H})], 8.22(\mathrm{~s}, 1 \mathrm{H}, \mathrm{CH}=\mathrm{N})$, 
9.90 (s, 1H, OH). ${ }^{13} \mathrm{C}$ NMR (100 MHz, DMSO-d $\left.\mathrm{d}_{6}\right)$ $\delta(\mathrm{ppm}), 12.45,14.12,31.28,60.22,100.87$, $115.51,125.81,128.82,148.25,152.67,159.17$, 161.34, 165.27. MS(ESI-m/z): $(\mathrm{M}+1)^{+}:$320.02; Anal. for $\mathrm{C}_{15} \mathrm{H}_{17} \mathrm{~N}_{3} \mathrm{O}_{3} \mathrm{~S}$ (Mw 319.38). Found \% C 56.48; H 5.31; N 13.25. Calculated \% C 56.41; H 5.37; N 13.16 .

(E)-Ethyl 2-(2-(4-hydroxy-3-methoxybenzylidene)hydrazono)-3,4-dimethyl-2,3-dihydro-thiazole-5-carboxylate (5b). The solid obtained was washed with $\mathrm{H}_{2} \mathrm{O}$ and recrystallized (yield: $2.86 \mathrm{~g}$, 91\%); yellow solid, $\mathrm{mp} 169-170^{\circ} \mathrm{C}$ (from DMFEtOH-water, 1:4:1) IR (KBr) $\left(v_{\max }, \mathrm{cm}^{-1}\right), 3100$ $3470(\mathrm{OH}), 3053(\mathrm{Ar}-\mathrm{CH}), 2934$ (Aliph. $\mathrm{CH})$, $1655(\mathrm{C}=\mathrm{O}), 1579(\mathrm{C}=\mathrm{N}), 1266-1196(\mathrm{C}-\mathrm{O}-\mathrm{C})$, $1087\left(\mathrm{OCH}_{2}-\mathrm{CH}_{3}\right)$. ${ }^{1} \mathrm{H}$ NMR $(200 \mathrm{MHz}, \mathrm{DMSO}-$ $\left.\mathrm{d}_{6}\right) \delta(\mathrm{ppm}), 1.21-1.27\left(\mathrm{t}, 3 \mathrm{H}\right.$, Ester- $\left.\mathrm{CH}_{3}\right), 2.51$ (s, $3 \mathrm{H}$, Thiazole- $\left.\mathrm{CH}_{3}\right), 3.39\left(\mathrm{~s}, 3 \mathrm{H}, \mathrm{N}-\mathrm{CH}_{3}\right), 3.81$ (s, $3 \mathrm{H}, \mathrm{OCH}_{3}$ ), 4.15-4.21 (q, 2H, Ester- $-\mathrm{OCH}_{2}$ ), Ar-H [6.80-6.84 (d, 1H)], 7.14-7.18 (d, 1H), $7.29(\mathrm{~s}$, $1 \mathrm{H})], 8.21(\mathrm{~s}, 1 \mathrm{H}, \mathrm{CH}=\mathrm{N}), 9.49(\mathrm{~s}, 1 \mathrm{H}, \mathrm{OH}) \cdot{ }^{13} \mathrm{C}$ NMR (100 MHz, DMSO-d $\left.{ }_{6}\right) \delta(\mathrm{ppm}), 12.48$, $14.20,31.35,55.42,60.27,100.48,109.93,115.48$, $121.44,126.23,147.70,148.27,148.68,152.99$, 161.37, 165.13. MS(ESI-m/z): $(\mathrm{M}+1)^{+}: 350.05$. Anal. for $\mathrm{C}_{16} \mathrm{H}_{19} \mathrm{~N}_{3} \mathrm{O}_{4} \mathrm{~S}$ (Mw 349.40). Found \% C 55.02; H 5.51; N 12.06. Calculated \% C 55.00; H $5.48 ; \mathrm{N} 12.03$.

(E)-Ethyl 2-(2-(4-hydroxy-3,5-dimethylbenzylidene)hydrazono)-3,4-dimethyl-2,3-dihydro-thiazole5-carboxylate $(\mathbf{5 c})$. The solid obtained was washed with EtOH then diethyl ether, and was recrystallized (yield: $2.22 \mathrm{~g}, 71 \%$ ); light-yellow solid, mp 203$204^{\circ} \mathrm{C}$ (from DMF-EtOH, 1:5) IR (KBr) $\left(v_{\max }, \mathrm{cm}^{-1}\right)$, $3437(\mathrm{OH}), 3120(\mathrm{Ar}-\mathrm{CH}), 2976$ (Aliph. CH), 1672 $(\mathrm{C}=\mathrm{O}), 1585 \quad(\mathrm{C}=\mathrm{N}), \quad 1293-1189 \quad(\mathrm{C}-\mathrm{O}-\mathrm{C}), 1093$ $\left(\mathrm{OCH}_{2}-\mathrm{CH}_{3}\right) .{ }^{1} \mathrm{H}$ NMR $\left(200 \mathrm{MHz}, \mathrm{DMSO}-\mathrm{d}_{6}\right) \delta$ (ppm), 1.26 (s, 3H, Ester- $\left.\mathrm{CH}_{3}\right), 2.21\left(\mathrm{~s}, 6 \mathrm{H}, \mathrm{CH}_{3}\right)$, $2.53\left(\mathrm{~s}, 3 \mathrm{H}\right.$, Thiazole- $\left.\mathrm{CH}_{3}\right), 3.38\left(\mathrm{~s}, 3 \mathrm{H}, \mathrm{N}-\mathrm{CH}_{3}\right)$, 4.20 (s, 2H, Ester- $\mathrm{OCH}_{2}$ ), Ar-H [7.31 (s, 2H)], 8.17 $(\mathrm{s}, 1 \mathrm{H}, \mathrm{CH}=\mathrm{N}), 8.71(\mathrm{~s}, 1 \mathrm{H}, \mathrm{OH}) .{ }^{13} \mathrm{C}$ NMR $(100$ MHz, DMSO-d $\left.\mathrm{d}_{6}\right) \delta(\mathrm{ppm}), 12.41,14.10,16.31$, $31.25,60.17,100.70,124.27,125.67,127.41$, 148.21, 153.18, 155.14, 161.31, 164.88. MS(ESI$m / z):(\mathrm{M}+1)^{+}:$348.12. Anal. for $\mathrm{C}_{17} \mathrm{H}_{21} \mathrm{~N}_{3} \mathrm{O}_{3} \mathrm{~S}(\mathrm{Mw}$ 347.43). Found \% C 58.69; H 6.18; N 12.12. Calculated \% C 58.77; H 6.09; N 12.09.

\section{General method for the synthesis of nitrile derivatives $(7 a-e)$}

In a two-necked flask, compounds 5a-e $(0.01 \mathrm{~mol})$ and $\mathrm{K}_{2} \mathrm{CO}_{3}(0.015 \mathrm{~mol})$ were dissolved in dry acetone, and the solution was stirred for 1 hour at room temperature. Compound 6 was then dropped into this solution. The reaction mixture was refluxed for 2 days. The solution was filtrated and the solid was obtained. The precipitated solid was recrystallized from an appropriate solvent to afford the desired compound.

(E)-Ethyl 2-(2-(4-(cyanomethoxy)benzylidene) hydrazono)-3,4-dimethyl-2,3-dihydro-thiazole-5-carboxylate (7a). The solid obtained was washed with acetone and recrystallized (yield: $3.01 \mathrm{~g}, 84 \%$ ); yellow crystals, mp $194-195^{\circ} \mathrm{C}$ (from DMFacetone, 1:3) IR (KBr) $\left(v_{\max }, \mathrm{cm}^{-1}\right), 3051(\mathrm{Ar}-\mathrm{CH})$, 2972 (Aliph. $\mathrm{CH}), 2255(\mathrm{C} \equiv \mathrm{N}), 1673(\mathrm{C}=\mathrm{O}), 1597$ $(\mathrm{C}=\mathrm{N}), 1289-1231$ (C-O-C), $1089\left(\mathrm{OCH}_{2}-\mathrm{CH}_{3}\right)$. ${ }^{1} \mathrm{H}$ NMR (200 MHz, DMSO-d $\left.{ }_{6}\right) \delta$ (ppm), 1.19$1.26\left(\mathrm{t}, 3 \mathrm{H}\right.$, Ester- $\left.\mathrm{CH}_{3}\right), 2.53(\mathrm{~s}, 3 \mathrm{H}$, Thiazole$\mathrm{CH}_{3}$ ), 3.37 (s, 3H, N-CH 3 ), 4.12-4.21 (q, 2H, Ester- $\left.\mathrm{OCH}_{2}\right), 5.21\left(\mathrm{~s}, 2 \mathrm{H}, \mathrm{Ar}-\mathrm{OCH}_{2}\right)$, Ar-H [7.09$7.14(\mathrm{~d}, 2 \mathrm{H}), 7.70-7.75, \mathrm{~d}, 2 \mathrm{H})], 8.51(\mathrm{~s}, 1 \mathrm{H}$, $\mathrm{CH}=\mathrm{N}) .{ }^{13} \mathrm{C}$ NMR $(100 \mathrm{MHz}$, DMSO-d 6 ) $\delta(\mathrm{ppm})$, 13.26, 14.90, 32.16, 54.19, 61.12, 101.94, 115.77, $117.19,129.49,130.01,149.06,152.56,158.19$, 162.08, 167.06. MS(ESI-m/z): $(\mathrm{M}+1)^{+}: \quad 359.02$. Anal. for $\mathrm{C}_{17} \mathrm{H}_{18} \mathrm{~N}_{4} \mathrm{O}_{3} \mathrm{~S}$ (Mw 358.41). Found \% C 56.92; H 5.03; N 15.68. Calculated \% C 56.97; H 5.06; N 15.63.

(E)-Ethyl 2-(2-(4-(cyanomethoxy)-3-methoxybenzylidene)hydrazono)-3,4-dimethyl-2,3-dihydro-

thiazole-5-carboxylate (7b). The solid obtained was washed with acetone and recrystallized (yield: $2.45 \mathrm{~g}, 63 \%$ ); white solid, $\mathrm{mp} 184-185^{\circ} \mathrm{C}$ (from acetone-chloroform, 3:1) IR (KBr) $\left(v_{\max }, \mathrm{cm}^{-1}\right)$, 3073 (Ar-CH), 2972 (Aliph. $\mathrm{CH}), 2245(\mathrm{C} \equiv \mathrm{N})$, $1689(\mathrm{C}=\mathrm{O}), 1582(\mathrm{C}=\mathrm{N}), 1268-1146(\mathrm{C}-\mathrm{O}-\mathrm{C})$, $1085\left(\mathrm{OCH}_{2}-\mathrm{CH}_{3}\right) .{ }^{1} \mathrm{H}$ NMR $(200 \mathrm{MHz}, \mathrm{DMSO}-$ $\left.\mathrm{d}_{6}\right) \delta(\mathrm{ppm}), 1.21-1.27\left(\mathrm{t}, 3 \mathrm{H}\right.$, Ester- $\left.\mathrm{CH}_{3}\right), 2.52(\mathrm{~s}$, $3 \mathrm{H}$, Thiazole- $\left.\mathrm{CH}_{3}\right), 3.36\left(\mathrm{~s}, 3 \mathrm{H}, \mathrm{N}-\mathrm{CH}_{3}\right), 3.84(\mathrm{~s}$, $\left.3 \mathrm{H}, \mathrm{OCH}_{3}\right), 4.13-4.23\left(\mathrm{q}, 2 \mathrm{H}\right.$, Ester- $\left.\mathrm{OCH}_{2}\right), 5.17$ (s, 2H, Ar-OCH ${ }_{2}$, Ar-H [7.13-7.17 (d, 1H), 7.287.33, d, 1H), 7.40, s, 1H)], $8.28(\mathrm{~s}, 1 \mathrm{H}, \mathrm{CH}=\mathrm{N}) .{ }^{13} \mathrm{C}$ NMR (100 MHz, DMSO-d $)_{6} \delta(\mathrm{ppm}), 13.31$, 14.93, 32.18, 55.09, 56.26, 61.12, 102.04, 110.68, $115.52,116.71,121.31,130.90,147.73,148.99$, $150.21,152.91,162.11,166.94$. MS(ESI $-m / z)$ : $(\mathrm{M}+1)^{+}$: 389.10. Anal. for $\mathrm{C}_{18} \mathrm{H}_{20} \mathrm{~N}_{4} \mathrm{O}_{4} \mathrm{~S}$ (Mw 388.44). Found \% C 55.52; H 5.12; N 14.40. Calculated \% C 55.66; H 5.19; N 14.42.

(E)-Ethyl 2-(2-(4-(cyanomethoxy)-3,5-dimethylbenzylidene)hydrazono)-3,4-dimethyl-2,3-dihydrothiazole-5-carboxylate (7c). The solid obtained was recrystallized (yield: $3.17 \mathrm{~g}, 82 \%$ ); yellow crystals, $\mathrm{mp} 174-175^{\circ} \mathrm{C}$ (from petroleum ether-chloroform, 1:1) IR (KBr) $\left(v_{\max }, \mathrm{cm}^{-1}\right), 3046$ (Ar-CH), 2979 (Aliph. CH), $2269(\mathrm{C} \equiv \mathrm{N}), 1691$ $(\mathrm{C}=\mathrm{O}), 1588(\mathrm{C}=\mathrm{N}), 1262-1159$ (C-O-C), 1081 
$\left(\mathrm{OCH}_{2}-\mathrm{CH}_{3}\right) .{ }^{1} \mathrm{H}$ NMR $\left(200 \mathrm{MHz}\right.$, DMSO-d $\left.\mathrm{d}_{6}\right) \delta$ (ppm), 1.20-1.27 (t, 3H, Ester- $\left.\mathrm{CH}_{3}\right), 2.28(\mathrm{~s}, 3 \mathrm{H}$, $\left.\mathrm{CH}_{3}\right), 2.52\left(\mathrm{~s}, 3 \mathrm{H}\right.$, Thiazole- $\left.\mathrm{CH}_{3}\right), 3.39(\mathrm{~s}, 3 \mathrm{H}, \mathrm{N}-$ $\left.\mathrm{CH}_{3}\right), 4.14-4.22$ (q, 2H, Ester- $\left.\mathrm{OCH}_{2}\right), 4.94$ (s, 2H, $\left.\mathrm{Ar}-\mathrm{OCH}_{2}\right), \mathrm{Ar}-\mathrm{H}[7.42(\mathrm{~s}, 2 \mathrm{H})], 8.22(\mathrm{~s}, 1 \mathrm{H}$, $\mathrm{CH}=\mathrm{N}) .{ }^{13} \mathrm{C}$ NMR $\left(100 \mathrm{MHz}, \mathrm{DMSO}-\mathrm{d}_{6}\right) \delta(\mathrm{ppm})$, $13.29,14.96,16.82,32.22,58.03,61.16,101.98$, $117.81,124.27,128.47,131.74,149.13,152.72$, 156.22, 162.09, 167.18. MS(ESI- $m / z):(\mathrm{M}+1)^{+}$: 387.04. Anal. for $\mathrm{C}_{19} \mathrm{H}_{22} \mathrm{~N}_{4} \mathrm{O}_{3} \mathrm{~S}$ (Mw 386.47). Found \% C 59.17; H 5.82; N 14.49. Calculated \% C 59.05; H 5.74; N 14.50 .

(E)-Ethyl 2-(2-(4-(cyanomethoxy)-3,5-dimethoxybenzylidene)hydrazono)-3,4-dimethyl-2,3-dihydrothiazole-5-carboxylate (7d). The solid obtained was recrystallized (yield: $3.01 \mathrm{~g}, 72 \%$ ); white solid, $\mathrm{mp} 220-221^{\circ} \mathrm{C}$ (from acetone) IR $(\mathrm{KBr})\left(v, \mathrm{~cm}^{-1}\right), 3068(\mathrm{Ar}-\mathrm{CH}), 2972$ (Aliph. $\left.\mathrm{CH}\right)$, $2243(\mathrm{C} \equiv \mathrm{N}), 1665(\mathrm{C}=\mathrm{O}), 1579(\mathrm{C}=\mathrm{N}), 1302-1240$ (C-O-C), $1091\left(\mathrm{OCH}_{2}-\mathrm{CH}_{3}\right) ;{ }^{1} \mathrm{H}$ NMR $(200 \mathrm{MHz}$, DMSO-d $\left.\mathrm{d}_{6}\right) \delta(\mathrm{ppm}), 1.25$ (s, 3H, Ester- $\left.\mathrm{CH}_{3}\right), 2.49$ (s, 3H, Thiazole- $\left.\mathrm{CH}_{3}\right), 3.41\left(\mathrm{~s}, 3 \mathrm{H}, \mathrm{N}-\mathrm{CH}_{3}\right)(3.84$ (s, 6H, $\left.\mathrm{OCH}_{3}\right), 4.17-4.24$ (s, 2H, Ester- $\mathrm{OCH}_{2}$ ), 4.92 (s, 2H, Ar- $\mathrm{OCH}_{2}$ ), Ar-H [7.10 (s, 2H)], 8.28 $(\mathrm{s}, 1 \mathrm{H}, \mathrm{CH}=\mathrm{N}) ;{ }^{13} \mathrm{C}$ NMR $\left(100 \mathrm{MHz}\right.$, DMSO-d $\left.\mathrm{d}_{6}\right) \delta$ (ppm), 13.31, 14.91, 32.81, 55.29, 56.54, 61.32, $102.28,110.84,115.32,116.91,117.75,121.51$, $130.55,146.54,147.95,150.35,152.19,162.25$, 167.25. MS(ESI- $/ z / z):(M+1)^{+}: 419.29$. Anal. for $\mathrm{C}_{19} \mathrm{H}_{22} \mathrm{~N}_{4} \mathrm{O}_{5} \mathrm{~S}$ (Mw 418.47). Found \% C 54.50; $\mathrm{H}$ 5.35; N 13.44. Calculated \% C 54.53; H 5.30; N 13.39 .

(2Z,2E)-Ethyl 2-(2-(2-(cyanomethoxy)benzylidene)hydrazono)-3,4-dimethyl-2,3-dihydrothiazole-5-carboxylate (7e). The solid obtained was recrystallized, (yield: $2.08 \mathrm{~g}, 58 \%$ ); brownish solid, mp $195-196^{\circ} \mathrm{C}$ (from petroleum ether-chloroformacetone, 1:1:1) IR $(\mathrm{KBr})\left(v_{\max }, \mathrm{cm}^{-1}\right), 3073(\mathrm{Ar}-$ $\mathrm{CH}), 2978$ (Aliph. $\mathrm{CH}), 2248(\mathrm{C} \equiv \mathrm{N}), 1676(\mathrm{C}=\mathrm{O})$, $1597(\mathrm{C}=\mathrm{N}), 1265-1169$ (C-O-C), $1088\left(\mathrm{OCH}_{2^{-}}\right.$ $\left.\mathrm{CH}_{3}\right) .{ }^{1} \mathrm{H}$ NMR $\left(200 \mathrm{MHz}\right.$, DMSO-d $\left.{ }_{6}\right) \delta(\mathrm{ppm})$, 1.19-1.27 (t, 3H, Ester- $\left.\mathrm{CH}_{3}\right), 2.53$ (s, 3H, Thiazole- $\left.\mathrm{CH}_{3}\right), 3.41\left(\mathrm{~s}, 3 \mathrm{H}, \mathrm{N}-\mathrm{CH}_{3}\right), 4.13-4.22(\mathrm{q}, 2 \mathrm{H}$, Ester- $\left.\mathrm{OCH}_{2}\right), 5.23$ (s, 2H, Ar-OCH$)_{2}, \mathrm{Ar}-\mathrm{H}$ [6.90$6.94(\mathrm{dd}, 1 \mathrm{H}), 7.13-7.22(\mathrm{dd}, 1 \mathrm{H}), 7.43-7.47(\mathrm{~d}$, 1H), 7.84-7.93 (m, 1H)], $8.28(\mathrm{~s}, 1 \mathrm{H}, \mathrm{CH}=\mathrm{N}) .{ }^{13} \mathrm{C}$ NMR (100 MHz, DMSO-d $\left.\mathrm{d}_{6}\right) \delta$ (ppm), 13.29, 14.90, 32.28, 54.83, 61.21, 102.13, 117.21, 114.09, $122.67,126.95,131.65,147.53,149.23,155.28$, 156.54, 162.19, 167.54. MS(ESI-m/z): $(\mathrm{M}+1)^{+}$: 359.09. Anal. for $\mathrm{C}_{17} \mathrm{H}_{18} \mathrm{~N}_{4} \mathrm{O}_{3} \mathrm{~S}$ (Mw 358.41). Found \% C 56.91; H 5.09; N 15.70. Calculated \% C 56.97; H 5.06; N 15.63.
General method for the synthesis of 2-amino-1,3,4-thiadiazole derivatives $(9 a-e)$

In a round-bottomed flask, a mixture of compounds $7 \mathbf{a}-\mathbf{e}(0.00553 \mathrm{~mol})$ and compound $\mathbf{8}$ $(0.00829 \mathrm{~mol})$ in trifluoroacetic acid $(5 \mathrm{ml})$ at 60 $70^{\circ} \mathrm{C}$ was stirred for $5 \mathrm{~h}$. The reaction mixture was poured into $200 \mathrm{ml}$ of ice-cold water and neutralized with ammonia. The solid obtained was washed with $\mathrm{H}_{2} \mathrm{O}$ and crystallized from an appropriate solvent to afford the desired compound.

(E)-Ethyl 2-(2-(4-((2-amino-1,3,4-thiadiazol-5-yl) methoxy)benzylidene)hydrazono)-3,4-dimethyl2,3-dihydrothiazole-5-carboxylate (9a). The solid obtained was recrystallized (yield: $1.36 \mathrm{~g}, 57 \%$ ); yellow solid, mp $256-257^{\circ} \mathrm{C}$ (from DMF-EtOH, 1:2) IR $(\mathrm{KBr})\left(v_{\max }, \mathrm{cm}^{-1}\right), 3313-3095\left(\mathrm{NH}_{2}\right), 3030$ (Ar-CH), 2982 (Aliph. CH), 1698 (C=O), 1601 $(\mathrm{C}=\mathrm{C}), 1577(\mathrm{~N}-\mathrm{C}-\mathrm{S}), 1540(\mathrm{C}=\mathrm{N}), 1088\left(\mathrm{OCH}_{2^{-}}\right.$ $\left.\mathrm{CH}_{3}\right), 1260-1170$ (C-O-C). ${ }^{1} \mathrm{H}$ NMR $(200 \mathrm{MHz}$, DMSO-d $\left.\mathrm{d}_{6}\right) \delta(\mathrm{ppm}), 1.24$ (s, 3H, Ester- $\left.\mathrm{CH}_{3}\right), 2.52$ (s, 3H, Thiazole- $\left.\mathrm{CH}_{3}\right), 3.36\left(\mathrm{~s}, 3 \mathrm{H}, \mathrm{N}-\mathrm{CH}_{3}\right), 4.80$ (s, $2 \mathrm{H}$, Ester- $\left.\mathrm{OCH}_{2}\right), 5.32$ (s, $\left.2 \mathrm{H}, \mathrm{Ar}-\mathrm{OCH}_{2}\right), \mathrm{Ar}-\mathrm{H}$ [7.10 (s, 2H), $7.69(\mathrm{~s}, 2 \mathrm{H})], 7.34\left(\mathrm{~s}, 2 \mathrm{H}, \mathrm{NH}_{2}\right), 8.27$ (s, $1 \mathrm{H}, \mathrm{CH}=\mathrm{N}) \cdot{ }^{13} \mathrm{C}$ NMR $\left(100 \mathrm{MHz}, \mathrm{DMSO}-\mathrm{d}_{6}\right) \delta$ (ppm), 13.30, 14.86, 31.22, 55.25, 60.16, 64.98, $100.65,109.41,112.55,120.65,129.42,146.75$, $151.24,152.86,153.92,163.76,167.25,170.55$. MS(ESI- $m / z): \quad(\mathrm{M}+1)^{+}:$433.24. Anal. for $\mathrm{C}_{18} \mathrm{H}_{20} \mathrm{~N}_{6} \mathrm{O}_{3} \mathrm{~S}_{2}$ (Mw 432.52). Found \% C 49.84; $\mathrm{H}$ 4.58; N 19.38. Calculated \% C 49.91; H 4.66; N 19.43 .

(E)-Ethyl 2-(2-(4-((2-amino-1,3,4-thiadiazol-5yl)methoxy)-3,5-dimethoxy)benzylidene)hydrazono)-3,4-dimethyl-2,3-dihydrothiazole-5-carboxylate (9b). The solid obtained was recrystallized (yield: $1.48 \mathrm{~g}, 58 \%$ ); yellow solid, $\mathrm{mp} 224-225^{\circ} \mathrm{C}$ (from DMF-EtOH, 1:1) IR (KBr) $\left(v_{\max }, \mathrm{cm}^{-1}\right)$, 3307-3121 $\left(\mathrm{NH}_{2}\right), 3034($ Ar- $\mathrm{CH}), 2986$ (Aliph. $\mathrm{CH}), 1676(\mathrm{C}=\mathrm{O}), 1577(\mathrm{C}=\mathrm{C}), 1546(\mathrm{~N}-\mathrm{C}-\mathrm{S})$, $1500(\mathrm{C}=\mathrm{N}), 1085-1132\left(\mathrm{OCH}_{2}-\mathrm{CH}_{3}\right), 1276-1227$ (C-O-C). ${ }^{1} \mathrm{H}$ NMR $\left(200 \mathrm{MHz}, \mathrm{DMSO}_{6}\right) \delta(\mathrm{ppm})$, $1.23\left(\mathrm{~s}, 3 \mathrm{H}\right.$, Ester- $\left.\mathrm{CH}_{3}\right), 2.52(\mathrm{~s}, 3 \mathrm{H}$, Thiazole$\mathrm{CH}_{3}$ ), 3.38 (s, 3H, N-CH $), 3.80$ (s, 3H), 4.19 (s, $2 \mathrm{H}$, Ester- $\left.\mathrm{OCH}_{2}\right), 5.30\left(\mathrm{~s}, 2 \mathrm{H}, \mathrm{Ar}-\mathrm{OCH}_{2}\right), \mathrm{Ar}-\mathrm{H}$ [7.18 (s, 1H), $7.26(\mathrm{~s}, 1 \mathrm{H}), 7.53(\mathrm{~s}, 1 \mathrm{H})], 7.34(\mathrm{~s}$, $\left.2 \mathrm{H}, \mathrm{NH}_{2}\right), 8.26(\mathrm{~s}, 1 \mathrm{H}, \mathrm{CH}=\mathrm{N}) .{ }^{13} \mathrm{C}$ NMR $(100$ $\left.\mathrm{MHz}, \mathrm{DMSO}-\mathrm{d}_{6}\right) \delta(\mathrm{ppm}), 13.32,14.96,32.21$, $56.25,61.16,65.58,101.85,110.41,114.85$, $121.66,129.52,148.95,149.16,150.04,153.26$, 154.52, 162.16, 166.64, 170.67. MS(ESI- $m / z)$ : $(\mathrm{M}+1)^{+}$: 463.08. Anal. for $\mathrm{C}_{19} \mathrm{H}_{22} \mathrm{~N}_{6} \mathrm{O}_{4} \mathrm{~S}_{2}$ (Mw 462.55). Found \% C 49.27; H 4.86; N 18.28. Calculated \% C 49.34; H 4.79; N 18.17 . 
(E)-Ethyl 2-(2-(4-((2-amino-1,3,4-thiadiazol-5yl)methoxy)-3,5-dimethyl-benzylidene)hydrazono)-3,4-dimethyl-2,3-dihydrothiazole-5-carboxylate $(9 \mathrm{c})$. The solid obtained was recrystallized (yield: $1.29 \mathrm{~g}, 51 \%$ ); light-yellow solid, mp 227$228^{\circ} \mathrm{C}$ (from DMF-EtOH, 1:8) IR (KBr) $\left(v_{\max }, \mathrm{cm}^{-1}\right)$, 3281-3131 $\left(\mathrm{NH}_{2}\right), 3043$ (Ar-CH), 2963 (Aliph. $\mathrm{CH}), 1688(\mathrm{C}=\mathrm{O}), 1583(\mathrm{C}=\mathrm{C}), 1536(\mathrm{~N}-\mathrm{C}-\mathrm{S})$, $1504(\mathrm{C}=\mathrm{N}), 1086\left(\mathrm{OCH}_{2}-\mathrm{CH}_{3}\right), 1266-1147(\mathrm{C}-\mathrm{O}-$ C). ${ }^{1} \mathrm{H}$ NMR $\left(200 \mathrm{MHz}, \mathrm{DMSO}-\mathrm{d}_{6}\right) \delta(\mathrm{ppm}), 1.20$ $1.26\left(\mathrm{t}, 3 \mathrm{H}\right.$, Ester- $\left.\mathrm{CH}_{3}\right), 2.23\left(\mathrm{~s}, 6 \mathrm{H}, \mathrm{CH}_{3}\right), 2.50(\mathrm{~s}$, $3 \mathrm{H}$, Thiazole- $\left.\mathrm{CH}_{3}\right), 3.37\left(\mathrm{~s}, 3 \mathrm{H}, \mathrm{N}-\mathrm{CH}_{3}\right), 4.14$ 4.19 (q, 2H, Ester- $-\mathrm{OCH}_{2}$ ), 4.97 (s, $2 \mathrm{H}, \mathrm{Ar}-\mathrm{OCH}_{2}$ ), Ar-H [7.34 (s, 2H)], 7.36 (s, 2H, NH $\mathrm{NH}_{2}, 8.19$ (s, 1H, $\mathrm{CH}=\mathrm{N}) .{ }^{13} \mathrm{C}$ NMR $(100 \mathrm{MHz}$, DMSO-d 6 ) $\delta(\mathrm{ppm})$, 13.26, 14.93, 16.91, 32.40, 61.13, 68.54, 101.95, $128.35,131.05,131.75,149.04,152.94,154.61$, 156.89, 162.10, 166.96, 170.68. MS(ESI-m/z): $(\mathrm{M}+1)^{+}$: 461.28. Anal. for $\mathrm{C}_{20} \mathrm{H}_{24} \mathrm{~N}_{6} \mathrm{O}_{3} \mathrm{~S}_{2}$ (Mw 460.57). Found \% C 52.28; H 5.19; N 18.30. Calculated \% C 52.16; H 5.25; N 18.25.

(E)-Ethyl 2-(2-(4-((2-amino-1,3,4-thiadiazol-5yl)methoxy)-3,5-dimethoxy-benzylidine)hydrazono)-3,4-dimethyl-2,3-dihydrothiazole-5-carboxylate (9d). The solid obtained was washed with $\mathrm{H}_{2} \mathrm{O}$ and recrystallized (yield: $1.55 \mathrm{~g}, 57 \%$ ); yellow solid, mp $211-212^{\circ} \mathrm{C}$ (from DMF-EtOH, 1:4) IR $(\mathrm{KBr})\left(v_{\max }, \mathrm{cm}^{-1}\right), 3297-3109\left(\mathrm{NH}_{2}\right), 3053(\mathrm{Ar}-$ $\mathrm{CH}), 2966$ (Aliph. $\mathrm{CH}), 1694(\mathrm{C}=\mathrm{O}), 1611(\mathrm{C}=\mathrm{C})$, 1581 (N-C-S), $1540(\mathrm{C}=\mathrm{N}), 1085-1121\left(\mathrm{OCH}_{2-}\right.$ $\left.\mathrm{CH}_{3}\right),{ }_{1264-1160}$ (C-O-C). ${ }^{1} \mathrm{H}$ NMR $(200 \mathrm{MHz}$, DMSO-d $\left.{ }_{6}\right) \delta(\mathrm{ppm}), 1.23$ (s, 3H, Ester- $\left.\mathrm{CH}_{3}\right), 2.51$ (s, 3H, Thiazole- $\left.\mathrm{CH}_{3}\right), 3.39\left(\mathrm{~s}, 3 \mathrm{H}, \mathrm{N}-\mathrm{CH}_{3}\right), 3.81$ (s, 6H, N-CH $\mathrm{CH}_{3}, 4.17-4.19$ (s, 2H, Ester- $\mathrm{OCH}_{2}$ ), 5.05 (s, 2H, Ar-OCH $\left.{ }_{2}\right)$, Ar-H [7.04 (s, 2H)], 7.34 (s, $\left.2 \mathrm{H}, \mathrm{NH}_{2}\right), 8.25(\mathrm{~s}, 1 \mathrm{H}, \mathrm{CH}=\mathrm{N}) .{ }^{13} \mathrm{C}$ NMR $(100$ MHz, DMSO-d $\left.\mathrm{d}_{6}\right) \delta(\mathrm{ppm}), 13.35,14.94,30.87$, $56.60,61.21,69.12,102.08,104.85,131.70$, $137.55,149.10,153.28,153.74,155.28,162.13$, 167.08, 170.87 MS(ESI-m/z): $(\mathrm{M}+1)^{+}: 493.08$. Anal. for $\mathrm{C}_{20} \mathrm{H}_{24} \mathrm{~N}_{6} \mathrm{O}_{5} \mathrm{~S}_{2}$ (Mw 492.57). Found \% C 48.89; H 4.99; N 17.13. Calculated \% C 48.77; H $4.91 ; \mathrm{N} 17.06$.

(2Z,2E)-Ethyl 2-(2-(2-((2-amino-1,3,4-thiadiazol-5yl)methoxy)-benzylidene)hydrazono)-3,4-dimethyl-2,3-dihydrothiazole-5-carboxylate (9e). The solid obtained was recrystallized (yield: $1.48 \mathrm{~g}$, $62 \%$ ); yellow solid, $\mathrm{mp} 247-248^{\circ} \mathrm{C}$ (from DMFEtOH, 1:8) IR (KBr) $\left(v_{\max }, \mathrm{cm}^{-1}\right), 3285-3169$ $\left(\mathrm{NH}_{2}\right), 3064$ (Ar-CH), 2977 (Aliph. $\left.\mathrm{CH}\right), 1672$ $(\mathrm{C}=\mathrm{O}), 1597(\mathrm{C}=\mathrm{C}), 1537(\mathrm{~N}-\mathrm{C}-\mathrm{S}), 1522(\mathrm{C}=\mathrm{N})$, $1081\left(\mathrm{OCH}_{2}-\mathrm{CH}_{3}\right), 1267-1228$ (C-O-C). ${ }^{1} \mathrm{H}$ NMR (200 MHz, DMSO-d $\left.\mathrm{d}_{6}\right) \delta(\mathrm{ppm}), 1.19-1.26(\mathrm{t}, 3 \mathrm{H}$, Ester- $\left.\mathrm{CH}_{3}\right), 2.51\left(\mathrm{~s}, 3 \mathrm{H}\right.$, Thiazole- $\left.\mathrm{CH}_{3}\right), 3.39(\mathrm{~s}$,
$3 \mathrm{H}, \mathrm{N}-\mathrm{CH}_{3}$ ), 4.13-4.20 (q, 2H, Ester- $\mathrm{OCH}_{2}$ ), 5.35 (s, 2H, Ar- $\left.\mathrm{OCH}_{2}\right), \mathrm{Ar}-\mathrm{H}$ [7.01-7.05 (m, 1H), 7.197.23, d, 1H), $7.35(\mathrm{~s}, 1 \mathrm{H}), 7.85-7.92(\mathrm{~s}, 1 \mathrm{H})], 7.34$ (s, $\left.2 \mathrm{H}, \mathrm{NH}_{2}\right), 8.50(\mathrm{~s}, 1 \mathrm{H}, \mathrm{CH}=\mathrm{N}) .{ }^{13} \mathrm{C}$ NMR $(100$ MHz, DMSO-d $\left.{ }_{6}\right) \delta$ (ppm), 13.26, 14.90, 32.26, $61.17,65.45,102.11,114.16,122.40,126.13$, $131.94,147.90,149.13,154.37,154.53,156.44$, 162.08, 167.61, 170.57. MS(ESI- $m / z)$ : $(\mathrm{M}+1)^{+}$: 433.24. Anal. for $\mathrm{C}_{18} \mathrm{H}_{20} \mathrm{~N}_{6} \mathrm{O}_{3} \mathrm{~S}_{2}$ (Mw 432.52). Found \% C 50.08; H 4.71; N 19.47. Calculated \% C 49.98; H 4.66; N 19.43.

\section{RESULTS AND DISCUSSION}

In the first part of the study, thiosemicarbazone derivatives 3a-e were synthesized via the reaction of various aldehyde derivatives $1 \mathbf{a}-\mathbf{e}$ with 4-methyl-thiosemicarbazide. In the IR spectral data of compounds 3a-e, signals belonging to the aldehyde carbonyl group that appeared at 1650-1730 $\mathrm{cm}^{-1}$ disappeared, and $v(\mathrm{NH})$ and $v(\mathrm{CH}=\mathrm{N})$ stretching frequencies were observed at 3147-3178 $\mathrm{cm}^{-1}$ and $1583-1599 \mathrm{~cm}^{-1}$, respectively. The presence of new absorption at $1583-1599 \mathrm{~cm}^{-1}$ belonging to $(\mathrm{CH}=\mathrm{N})$ supported the data indicating that the thiosemicarbazones derivatives $\mathbf{3 a}-\mathbf{e}$ were successfully prepared. Also, structures of the thiosemicarbazone derivatives 3a-e were identified with the assistance of NMR spectroscopy.

In the ${ }^{1} \mathrm{H}$ NMR spectra of compounds $\mathbf{3 a}-\mathbf{e}$, the proton signals were recorded at $8.34-8.37 \mathrm{ppm}$ $\left(\mathrm{NH}-\mathrm{CH}_{3}\right)$ integrating for one proton (controlled by exchanging with $\left.\mathrm{D}_{2} \mathrm{O}\right)$. $\mathrm{N}(2) \mathrm{H}$ protons were observed between 11.26 and $11.32 \mathrm{ppm}$ integrating for one proton (exchangeable with $\mathrm{D}_{2} \mathrm{O}$ ). In the ${ }^{1} \mathrm{H}$ NMR spectra of compounds 3a-e, another characteristic proton signal belonging to $\mathrm{CH}=\mathrm{N}$ was observed at 7.88-7.94 ppm integrating for one proton. In the ${ }^{1} \mathrm{H}$ NMR data of compounds $3 \mathbf{a}-\mathbf{e}$, the signals of the aldehyde protons and the $-\mathrm{NH}_{2}$ protons of 4-methyl-3-thiosemicarbazide disappeared and a new signal belonging to the protons of the $\mathrm{CH}=\mathrm{N}$ group was observed at 7.88-7.94 ppm. Furthermore, in the ${ }^{13} \mathrm{C}$ NMR data, the signal of carbon from the carbonyl group of aldehyde derivatives disappeared, and a new signal appeared at 142.04$143.20 \mathrm{ppm}$ belonging to the iminic carbon.

In the ${ }^{13} \mathrm{C}$ NMR spectral data of compounds 3a-e, while $\mathrm{C}=\mathrm{O}$ belonging to the ${ }^{13} \mathrm{C}$ NMR signal of compounds 1a-e that appeared at 185-195 ppm disappeared, the ${ }^{13} \mathrm{C}$ NMR signals belonging to the $\mathrm{C}=\mathrm{S}$ group of compounds $\mathbf{3 a}-\mathbf{e}$ were observed at 177.22-177.84 ppm. It has been reported that the peak of the spectral data is quite specific [28]. On 
the other hand, the mass spectra of compounds 3ae confirmed the structure of $\mathbf{3 a}-\mathbf{e}$ by molecular ion peaks.

In the second part of the study, the thiazole moieties of the target materials were obtained using the Hantzsch reaction from the reaction thiosemicarbazone derivatives $\mathbf{3 a}-\mathbf{e}$ with ethyl-2chloroacetoacetic ester at a 1:1 ratio in absolute ethanol in high yields (Scheme 1). According to this reaction mechanism, the compounds of $\mathbf{5 a - e}$ could be resonance forms. However, the spectral data and physical parameters showed that the compounds of $\mathbf{5 a}$-e have the exo-imine form in the 2position of the thiazole group. The reaction mechanism is shown in Scheme 2.<smiles>[X]c1cc(/C=N/NC(NC)SCCCCCCCC)cc([X])c1O</smiles><smiles>[X]c1cc(/C=N/N/N=[N+](/C)SC(C(C)=O)C(=O)OCC)cc([X])c1O</smiles>

3a-e

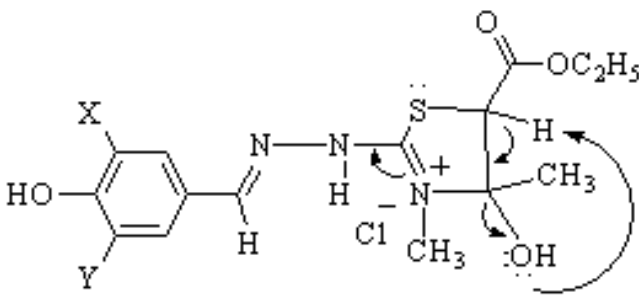<smiles>C=CC</smiles>

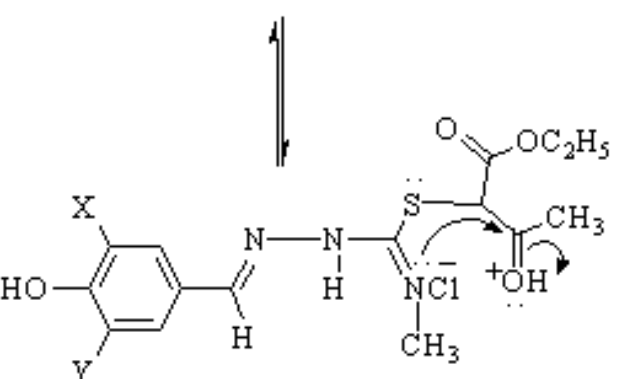<smiles>[X]c1cc(/C=N/N=c2/sc(C(=O)OCC)c(C)n2C)cc([X])c1O</smiles>

5a-e<smiles></smiles>

$5 a^{\prime}-e^{\prime}$

Scheme 2. The mechanism of the Hantzsch thiazole synthesis

The most characteristic IR data for the synthesized compounds 5a-e appeared at 1655-1700 $\mathrm{cm}^{-1}$ ( $\mathrm{C}=\mathrm{O}$ stretching), $1579-1603 \mathrm{~cm}^{-1}(\mathrm{CH}=\mathrm{N}$ stretching), and $1189-1293 \mathrm{~cm}^{-1}$ (C-O-C stretching). The absence of absorption of the $-\mathrm{NH}$ groups of compounds 3a-e in the IR spectra of compounds $\mathbf{5 a}-\mathbf{e}$ confirmed the realization of the reaction. Furthermore, the ${ }^{1} \mathrm{H}$ NMR spectra showed the absence of the $-\mathrm{N}(2) \mathrm{H}$ signals of the compounds 3a-e and the presence of the new methyl and ethoxy group signals at 3.37-3.39, 4.144.23 (for $\mathrm{OCH}_{2}$ ) and 1.20-1.28 ppm (for $\mathrm{CH}_{3}$ of ethoxy group), respectively. The ${ }^{1} \mathrm{H}$ NMR spectra of compounds $\mathbf{5 a}-\mathbf{e}$ also supported the proposed structure. The ${ }^{13} \mathrm{C}$ NMR spectra of $\mathbf{5 a}-\mathbf{e}$ provided satisfactory data for their characterization. The ${ }^{13} \mathrm{C}$ NMR signals of the compounds $\mathbf{5 a}-\mathbf{e}$ of the $-\mathrm{CH}_{3}$ and $-\mathrm{OCH}_{2}$ ester group and the carbons of the thiazole ring $-\mathrm{CH}_{3}$ and $-\mathrm{OCH}_{2}$ were observed at $12.41-$ $60.27 \mathrm{ppm}$. This spectral data provides the strongest evidence for $\mathrm{sp}^{3}$-hybridized carbons. In addition, $\mathrm{C}(5)$ and $\mathrm{C}(4)$ of the thiazole ring were observed at 100.48-100.87 and 148.21-148.28 ppm, respectively. The exo $\mathrm{N}\left(2^{\prime}\right)=\mathrm{C}(2)$ carbon data of the 
thiazole ring appeared at $161.31-161.37 \mathrm{ppm}$ in the ${ }^{13} \mathrm{C}$ NMR spectra [29]. The signals of the thione group $(\mathrm{C}=\mathrm{S})$ from the compounds $3 \mathbf{a}-\mathbf{e}$ disappeared, and new signals were observed at $60.17-$ $60.27 \mathrm{ppm}\left(-\mathrm{OCH}_{2}\right), 14.10-14.20 \mathrm{ppm}\left(-\mathrm{CH}_{3}\right)$, 12.41-12.54 ppm (-OCH $\left.\mathrm{CH}_{3}\right)$. On the other hand, the mass spectra of compounds $\mathbf{5 a}-\mathbf{e}$ supported the structure of $\mathbf{5 a}-\mathbf{e}$ by molecular ion peaks.

In the third part of the study, substituted nitrile derivatives $\mathbf{7 a}-\mathbf{e}$ were obtained from the reaction of compounds 5a-e with chloroacetonitrile (Scheme 1). The preparations of the substituted nitrile derivatives $\mathbf{7 a - e}$ were achieved by the nucleophilic aliphatic substitution reaction of chloroacetonitrile with compounds 5a-e [30]. The structures of compounds were confirmed by spectral investigation. In the IR spectra, the formations of compounds $\mathbf{7 a - e}$ were clearly defined by the disappearance of $-\mathrm{OH}$ absorption of compounds $\mathbf{5 a}-\mathbf{e}$, and the appearance of a $-\mathrm{C} \equiv \mathrm{N}$ absorption band between 2243 and $2269 \mathrm{~cm}^{-1}$. The ${ }^{1} \mathrm{H}$ NMR spectra of compounds 7a-e were also in good agreement with the structures of the synthesized compounds 7a-e. The phenolic -OH signal of compounds 5a-e disappeared after the nucleophilic aliphatic substitution reaction. The integral ratios of the aliphatic $\left(-\mathrm{CH}_{2}\right)$ proton signals were obtained as expected. The $-\mathrm{CH}_{2}$ protons of compounds $7 \mathbf{a}-\mathbf{e}$ were observed at 4.12-4.24 ppm integrating for two protons (exchangeable with $\mathrm{D}_{2} \mathrm{O}$ ). In the ${ }^{13} \mathrm{C}$ NMR spectra, the signals seen at 116.71-117.81 ppm indicated the presence of nitrile carbon for compounds 7a-e. Additionally, the mass spectra of compounds $7 \mathbf{a}-\mathbf{e}$ validated the structure of $7 \mathbf{a}-\mathbf{e}$ by molecular ion peaks.

In the last part of the study, substituted 2amino-1,3,4-thiadiazole compounds 9a-e were obtained from the reaction of thiosemicarbazide with substituted nitrile derivatives $7 \mathbf{a}-\mathbf{e}$ in the presence of trifluoroacetic acid (TFA) (Scheme 1). The reaction mechanism is shown in Scheme 3.

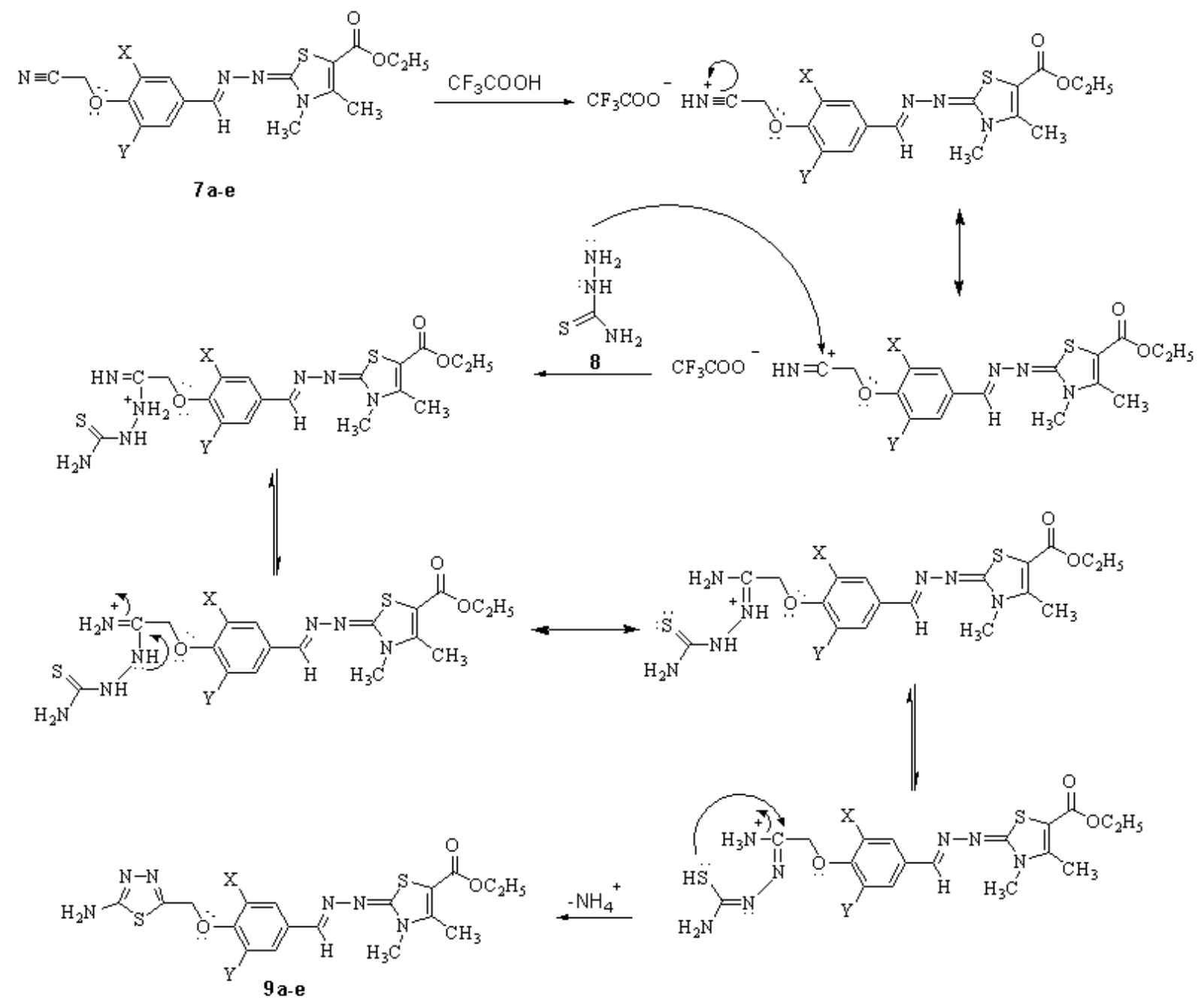

Scheme 3. The mechanism of the substituted 2-amino-1,3,4-thiadiazole synthesis. 
In the IR spectra of compounds $9 \mathbf{a}-\mathbf{e},-\mathrm{NH}_{2}$ was observed at $3131-3313 \mathrm{~cm}^{-1}$, and the C-O-C stretching frequency was observed at 1020-1132 $\mathrm{cm}^{-1}$. In the ${ }^{1} \mathrm{H}$ NMR spectra of compounds $9 \mathbf{a}-\mathbf{e}$, the proton signals from the methylene group $\left(-\mathrm{CH}_{2}\right)$ were recorded at $4.97-5.35 \mathrm{ppm}$ integrating for two protons. $-\mathrm{NH}_{2}$ was observed at $7.27-7.36 \mathrm{ppm}$ integrating for two protons (exchangeable with $\mathrm{D}_{2} \mathrm{O}$ ). In the ${ }^{13} \mathrm{C}$ NMR spectra of compounds $9 \mathbf{a}-\mathbf{e}$, the signals belonging to the thiadiazole ring $(\mathrm{C}-2$ and $\mathrm{C}-5)$ were observed in the aromatic region while the signal belonging to the $-\mathrm{C} \equiv \mathrm{N}$ group disappeared. The thiadiazole rings of compounds $9 \mathbf{a}-$ e were observed at $153.74-154.61 \mathrm{ppm}$ for $\mathrm{C}-2$ and 170.57-170.87 for C-5. The ${ }^{13} \mathrm{C}$ NMR signals of the methylene group were observed between 65.45 and $69.12 \mathrm{ppm}$. On the other hand, the mass spectra of compounds $9 \mathbf{a}-\mathbf{e}$ confirmed the structure of compounds $9 \mathbf{a}-\mathbf{e}$ by molecular ion peaks.

Generally, the presence of electron-donor groups led to increased product yields. However, in the presence of 2,6-disubstituted electron-donor groups, either low yields were obtained or the target compounds could not be obtained because of steric effects. Thus, in this study, 3,5-disubstituted electron-donor groups were used, and the obtained reaction yields were presented in experimental section.

In this study, novel thiosemicarbazone, 1,3thiazole, nitrile and 2-amino-1,3,4-thiadiazole derivatives were synthesized and characterized by IR, ${ }^{1} \mathrm{H}$ NMR, ${ }^{13} \mathrm{C}$ NMR, elemental analysis, and mass spectral analysis techniques. The methods used in this study are applicable for obtaining compounds with a strong potential for biological activity.

Acknowledgments. We thank Professor İ. Değirmencioğlu and Professor K. Serbest for their valuable contributions.

\section{REFERENCES}

[1] A. K. Jain, S. Sharma, A. Vaidya, V. Ravichandran, R. K. Agrawal, 1,3,4-Thiadiazole and its derivatives: A review on recent progress in biological activities, Chem. Biol. Drug. Des., 81, 557-576 (2013).

[2] G. Kolavi, V. Hedge, I. A. Khazi, Intramolecular amidation: Synthesis of novel imidazo[2,1-b][1,3,4]thiadiazole and imidazo[2,1-b][1,3]thiazole fused diazepinones, Tetrahedron Lett., 47, 2811-2814 (2006).

[3] J. M. Guernon, Y. J. Wu, 3-Bromocyclohexane-1,2dione as a useful reagent for Hantzsch synthesis of thiazoles and the synthesis of related heterocycles, Tetrahedron Lett., 52, 3633-3635 (2011).

[4] K. D. Hargrave, F. K. Hess, J. T. Oliver, N-(4substituted-thiazolyl)oxamic acid derivatives, a new series of potent, orally active antiallergy agents, J. Med. Chem., 26, 1158-1163 (1983).
[5] S. Bondock, H. El-Azab, E. E. M. Kandeel, M. A. Metwally, Efficient synthesis of new functionalized 2(hetaryl)thiazoles, Synth. Commun., 43, 59-71 (2013).

[6] A. A. Kadi, E. S. Al-Abdullah, I. A. Shehata, E. E. Habib, T. M. Ibrahim, A. A. El-Emam, Synthesis, antimicrobial and anti-inflammatory activities of novel 5-(1adamantyl)-1,3,4-thiadiazole derivatives, Eur. J. Med. Chem., 45, 5006-5011 (2010).

[7] S. K. Bhati, A. Kumar, Synthesis of new substituted azetidinoyl and thiazolidinoyl-1,3,4-thiadiazino (6,5-b) indoles as promising anti-inflammatory agents, Eur. $J$. Med. Chem., 43, 2323-2330 (2008).

[8] J. C. Jaen, L. D. Wise, B. W. Caprathe, H. Tecle, S. Bergmeier, C. C. Humblet, T. G. Heffner, L. T. Meltzner, T. A. Pugsley, 4-(1,2,5,6-Tetrahydro-1-alkyl3-pyridinyl)-2-thiazolamines: A novel class of compounds with central dopamine agonist properties, $J$. Med. Chem., 33, 311-317 (1990).

[9] J. Matysiak, Z. Malinski, 2-(2,4-Dihydroxyphenyl)1,3,4-thiadiazole analogues: Antifungal activity in vitro against candida species, Russ. J. Bioorg. Chem., 33, 594-601 (2007).

[10] A. A. Kadi, N. R. El-Brollosy, O. A. Al-Deeb, E. E. Habib, T. M. Ibrahim, A. A. El-Emam, Synthesis, antimicrobial and anti-inflammatory activities of novel 2-(1adamantyl)-5-substituted-1,3,4-oxadiazoles and 2-(1adamantylamino)-5-substituted-1,3,4-thiadiazoles, Eur. J. Med. Chem., 42, 235-242 (2007).

[11] S. N. Pandeya, D. Sriram, G. Nath, E. DeClerq, Synthesis, antibacterial, antifungal and anti-HIV activities of Schiff and Mannich bases derived from isatin derivatives and N-[4-(4'-chlorophenyl)thiazol-2-yl] thiosemicarbazide, Eur. J. Pharm. Sci., 9, 25-31 (1999).

[12] N. U. Güzeldemirci, Ö. Küçükbasmac1, Synthesis and antimicrobial activity evaluation of new 1,2,4-triazoles and 1,3,4-thiadiazoles bearing imidazo[2,1-b]thiazole moiety, Eur. J. Med. Chem., 45, 63-68 (2010).

[13] G. V. S. Kumar, Y. R. Prasad, B. P. Mallikarjuna, S. M. Chandrashekar, Synthesis and pharmacological evaluation of clubbed isopropylthiazole derived triazolothiadiazoles, triazolothiadiazines and mannich bases as potential antimicrobial and antitubercular agents, Eur. J. Med. Chem., 45, 5120-5129 (2010).

[14] S. A. F. Rostom, I. M. El-Ashmawy, H. A. B. El Razik, M. H. Badr, H. M. A. Ashour, Design and synthesis of some thiazolyl and thiadiazolyl derivatives of antipyrine as potential non-acidic anti-inflammatory, analgesic and antimicrobial agents, Bioorg. Med. Chem., 17, 882-895 (2009).

[15] P. Zhan, X. Liu, Z. Li, Z. Fang, Z. Li, D. Wang, C. Pannecouque, E. De Clercq, Novel 1,2,3-thiadiazole derivatives as HIV-1 NNRTIs with improved potency: Synthesis and preliminary SAR studies, Bioorg. Med. Chem., 17, 5920-5927 (2009).

[16] N. Siddiqui, W. Ahsan, Synthesis, anticonvulsant and toxicity screening of thiazolyl-thiadiazole derivatives, Med. Chem. Res., 20, 261-268 (2011).

[17] M. Yusuf, R. A. Khan, B. Ahmed, Syntheses and antidepressant activity of 5-amino-1, 3, 4-thiadiazole-2-thiol imines and thiobenzyl derivatives, Bioorg. Med. Chem., 16, 8029-8034 (2008). 
[18] M. Juszczak, J. Matysiak, M. Szeliga, P. Pozarowski, A Niewiadomy, J. Albrecht, W. Rzeski, 2-Amino-1,3,4thiadiazole derivative (FABT) inhibits the extracellular signal-regulated kinase pathway and induces cell cycle arrest in human non-small lung carcinoma cells, Bioorg. Med. Chem. Lett., 22, 5466-5469 (2012).

[19] K. M. Dawood, T. M. A. Eldebss, H. S. A. El-Zahabi, M. H. Yousef, P. Metz, Synthesis of some new pyrazolebased 1,3-thiazoles and 1,3,4-thiadiazoles as anticancer agents, Eur. J. Med. Chem., 70, 740-749 (2013).

[20] S. S. Karki, K. Panjamurthy, S. Kumar, M. Nambiar, S. A. Ramareddy, K. K. Chiruvella, S. C. Raghavan, Synthesis and biological evaluation of novel 2-aralkyl-5substituted-6-(4'-fluorophenyl)-imidazo[2,1-b][1,3,4]thiadiazole derivatives as potent anticancer agents, Eur. $J$. Med. Chem., 46, 2109-2116 (2011).

[21] I. Kayagil, S. Demirayak, Synthesis and anticancer activities of some thiazole derivatives, Phosphorus, Sulfur Silicon Relat. Elem., 184, 2197-2207 (2009).

[22] F. Karipcin, B. Dede, S. Percin-Ozkorucuklu, E. Kabalcilar, $\mathrm{Mn}(\mathrm{II}), \mathrm{Co}$ (II) and $\mathrm{Ni}(\mathrm{II})$ complexes of 4-(2thiazolylazo)resorcinol: Syntheses, characterization, catalase-like activity, thermal and electrochemical behavior, Dyes and Pigments, 84, 14-18 (2010).

[23] F. Clemence, O. L. Martert, F. Delevallee, J. Benzoni, A. Jouanen, S. Jouquey, M. Mouren, R. Deraedt, 4-Hydroxy3-quinolinecarboxamides with antiarthritic and analgesic activities, J. Med. Chem., 31, 1453-1462 (1998).

[24] J. V. Metzger, Comprehensive Heterocyclic Chemistry 1; Pergamon Press, Vol. 6, 1984, p 328.
[25] B. B. Shingate, B. G. Hazra, D. B. Salunke, V. S. Pore, F. Shirazi, M. V. Deshpande, Stereoselective synthesis and antimicrobial activity of steroidal C-20 tertiary alcohols with thiazole/pyridine side, J. Med. Chem., 46, 3681-3689 (2011).

[26] G. Dede, R. Bayrak, M. Er. A. R. Özkaya, İ. Değirmencioğlu, DBU-catalyzed condensation of metal free and metallophthalocyanines containing thiazole and azine moieties-synthesis, characterization and electrochemical properties, J. Organomet. Chem., 740, 70-77 (2013).

[27] İ. Değirmencioğlu, E. Atalay, M. Er, Y. Köysal, Ş. Işık, K. Serbest, Novel phthalocyanines containing substituted salicyclic hydrazone-1,3-thiazole moieties: Microwave-assisted synthesis, spectroscopic characterization, X-ray structure and thermal characterization, Dyes and Pigments, 84, 69-78 (2009).

[28] M. Er, Y. Ünver, K. Sancak, E. Dügdü, Synthesis and characterizations of some new tetrathiosemicarbazones and their cyclization reactions; tetra-4-methyl-5-etoxycarbonyl-2,3-dihydro-1,3-thiazole and tetra-2-acetylamino-4-acetyl-4,5-dihydro-1,3,4-thiodiazole derivatives, Arkivoc, (xv), 99-120 (2008).

[29] M. D. Mullican, M. W. Wilson, D. T. Connor, C. R. Konstlan, D. J. Schrier, R. D. Dyer, Design of 5-(3,5-ditert-butyl-4-hydroxyphenyl)-1,3,4-thiadiazoles, -1,3,4-oxadiazoles, and -1,2,4-triazoles as orally-active, nonulcerogenic antiinflammatory agents, J. Med. Chem., 36, 1090-1099 (1993).

[30] R. Ustabaș, U. Çoruh, K. Sancak, M. Er, Y. Ünver, M. Yavuz, 2-[2-(Cyanomethoxy) phenoxy]acetonitrile, Acta Crytallographica, Sect. E, 60, 968-970 (2004). 